\title{
Geometric Orbits
}

\section{Maria Carvalho \& Mika Hager}

The Mathematical Intelligencer

ISSN 0343-6993

Volume 34

Number 2

Math Intelligencer (2012) 34:56-62

DOI 10.1007/s00283-012-9287-y

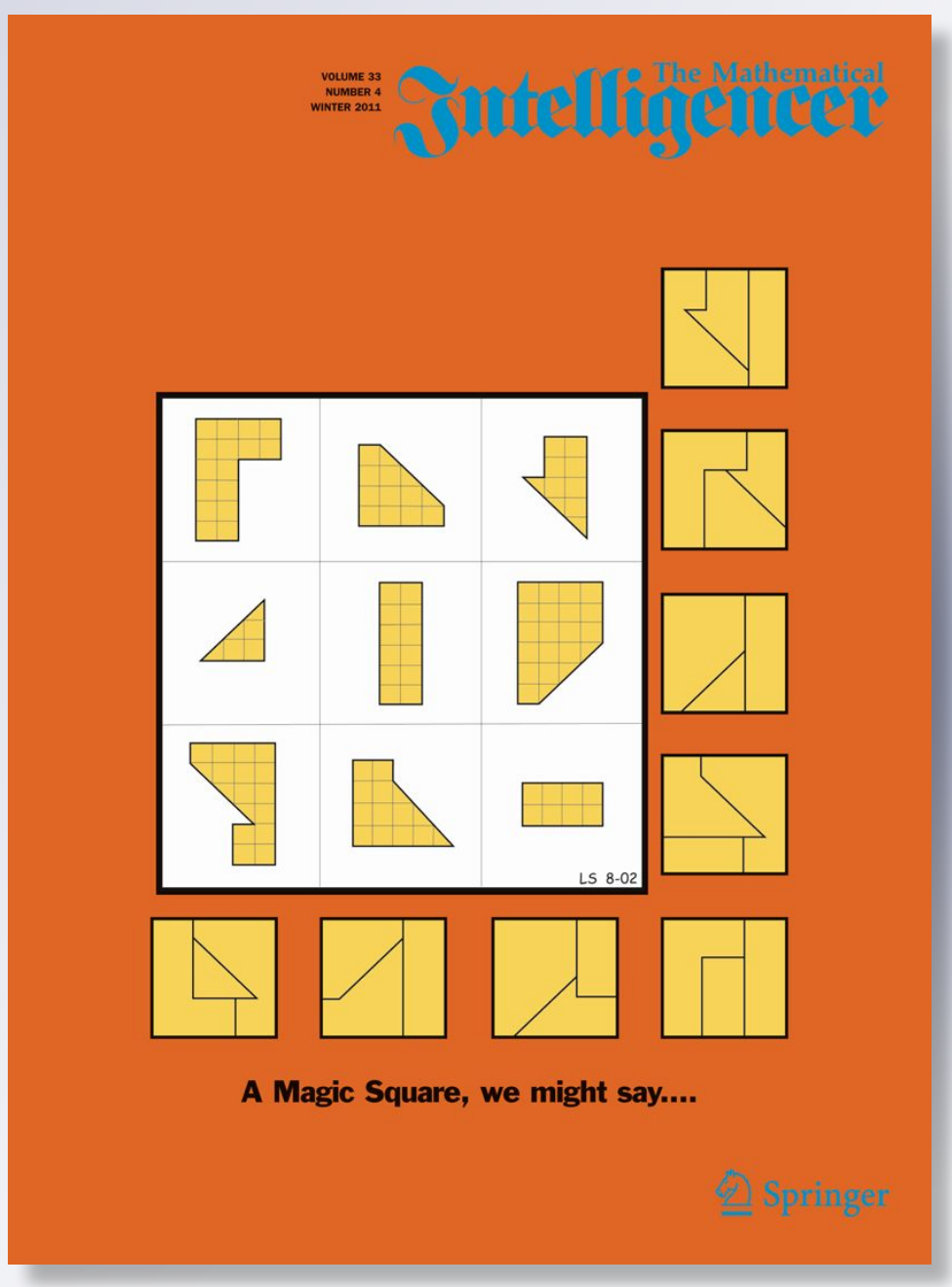

望 Springer 
Your article is protected by copyright and all rights are held exclusively by Springer Science+Business Media, LLC. This e-offprint is for personal use only and shall not be selfarchived in electronic repositories. If you wish to self-archive your work, please use the accepted author's version for posting to your own website or your institution's repository. You may further deposit the accepted author's version on a funder's repository at a funder's request, provided it is not made publicly available until 12 months after publication. 


\section{Geometric Orbits}

\section{Maria Carvalho* and Mika Hager ${ }^{\dagger}$}

1 $n$ the year 2006, the award of the eleventh edition of the Pirelli International Prize in the category of Science Communication of Mathematics was granted to a multimedia work created by David Gale at the University of California, Berkeley, USA. The jury stressed how efficiently Gale's contribution provided the wider public with an informal and interactive approach to complex mathematics. As soon as the winner was announced, we hurried to know more about the laureate's website. ${ }^{1}$ Enticed by the section concerning geometry and dynamical systems, ${ }^{2}$ we started tracing orbits and guessing their asymptotic behaviour. This is an account of that experience, with proofs of the properties we found.

Consider, on the plane, a triangle with sides labelled $a, b, c$, a point $P$ and its trajectory as it visits each side of the triangle in alphabetical order, always taking the shortest route.
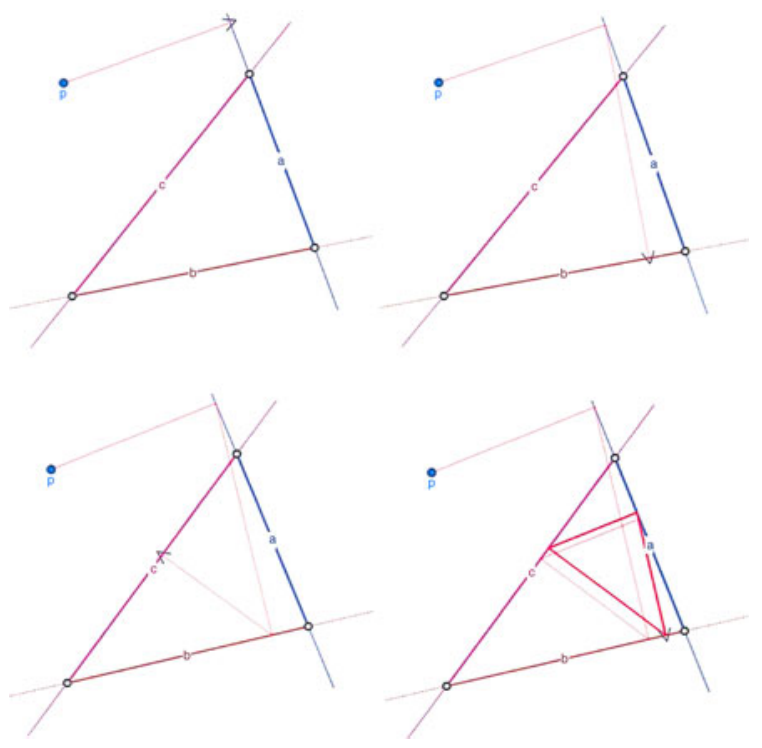

We may iterate this process, and then repeat it after changing either the initial point
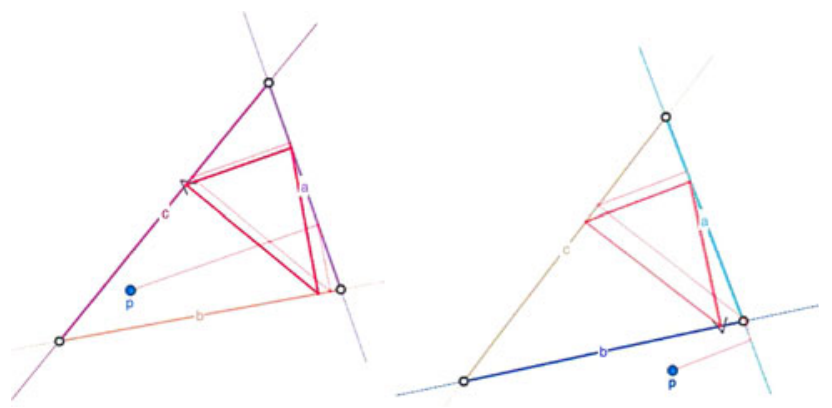

or the triangle.

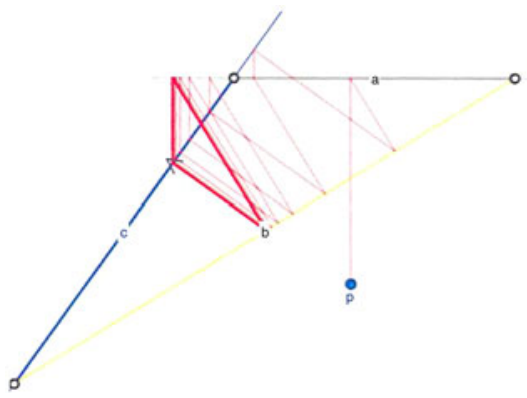

After inspecting a few orbits in a number of different triangles, we notice that, for each triangle, there is a limit figure, which is another triangle that does not depend on the choice of the starting point. Looking more closely, this limit triangle seems similar to the original one, although rotated by 90 degrees. Moreover, if we reverse the order in which the trajectories touch the sides of the triangle, from $a, b, c$ to $b, a, c$, then the limit triangles (the red one for $a, b, c$, the blue one for $b, a, c)$ look congruent, with the six vertices lying on the same circle.

*Partially supported by FCT through CMUP.

†Supported by Calouste Gulbenkian Foundation through the Program Novos Talentos em Matemática.

${ }^{1} \mathrm{http}: / /$ mathsite.math.berkeley.edu/main.html

${ }^{2}$ http://mathsite.math.berkeley.edu/geometricOrbits/geometricOrbits.html 

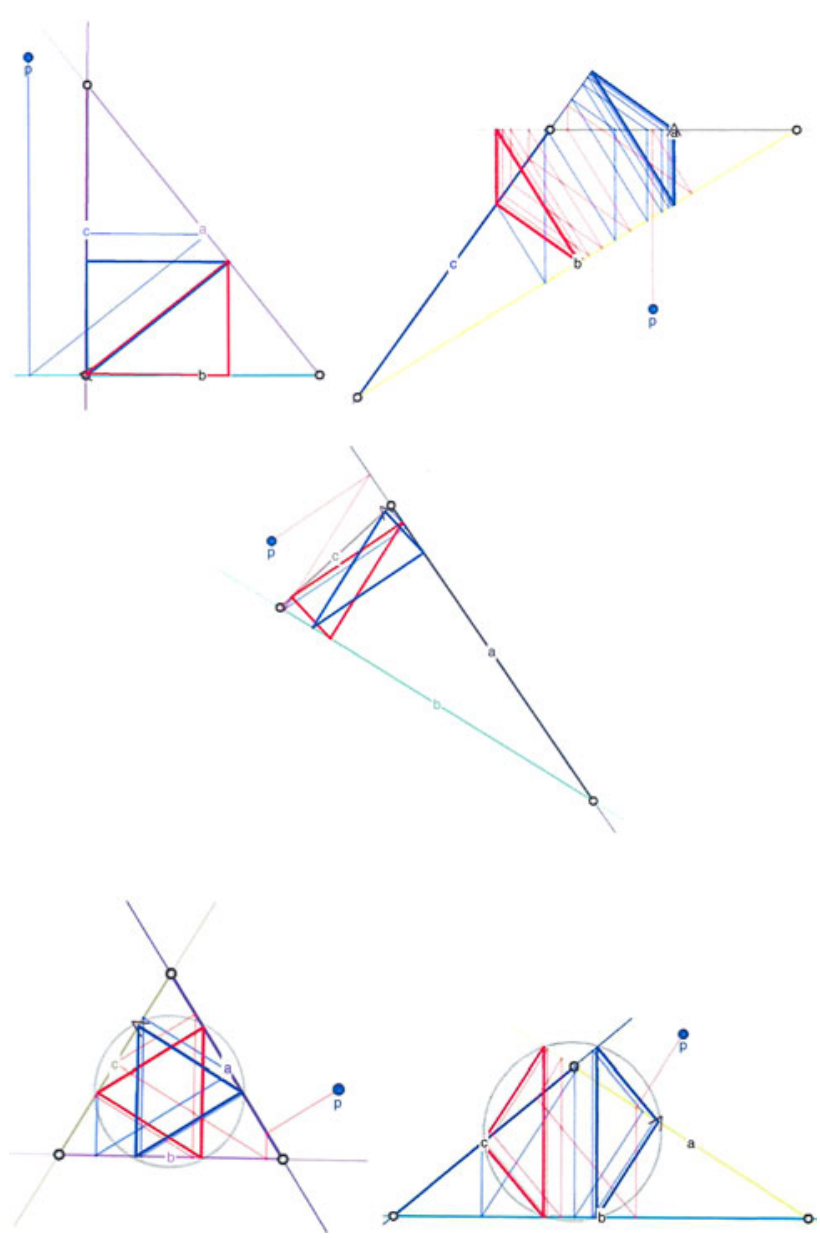

To prove these and other properties the experiments suggest, we formalized this iterative process as a mapping of one side of the triangle into itself. After this was done, we analysed a more general setting where the sides of the fixed triangle are visited through routes that may not be the shortest ones and may not respect the alphabetical order.

\section{Dynamical Systems}

A discrete dynamical system on a space $\mathcal{X}$ is a function $\mathcal{F}: \mathbb{N}_{0} \times \mathcal{X} \rightarrow \mathcal{X}$ defined by $\mathcal{F}(n, x)=\phi^{n}(x)$, where $\phi:$ $\mathcal{X} \rightarrow \mathcal{X}$ is a map, $\phi^{0}=$ Identity $_{\mathcal{X}}$ and $\phi^{n}$ is the composition of $\phi$ with itself $n$ times. In this way, we assign to each $x \in \mathcal{X}$ a sequence $\left(\phi^{n}(x)\right)_{n \in \mathbb{N}_{0}}$, called the orbit of $x$ by $\phi$. In the study of $\mathcal{F}$, we aim at a complete description of the set of accumulation points of the orbit of each $x$ (the so called $\omega$-limit of $x$ ) and how it varies with the initial point $x$.

For instance, if $\phi(x)=x, x \in \mathbb{R}$, then each point has the simplest possible orbit, the constant sequence equal to $x$. If $\phi(x)=1-x, x \in \mathbb{R}$, then there is a fixed point, $x=\frac{1}{2}$, and all other orbits are periodic with period two (that is, if $x \neq \frac{1}{2}$, then $\phi(x) \neq x$ and $\left.\phi \circ \phi(x)=x\right)$. If $\phi(x)=x^{2}, x \in$ $[0,1]$, then there are two fixed points, $x=0$ and $x=1$, and all the other orbits converge to 0 , which is appropriately called an attracting fixed point. If $\left.\phi(x)=1+\frac{1}{x}, x \in\right] 0, \infty[$, then again $\phi$ is a contraction on a neighbourhood of its unique fixed point, $\frac{1+\sqrt{5}}{2}$, and all the other orbits approach it, although not monotonically. (In particular, this is true for the orbit of 1 , whose elements are the sequence of the ratios of consecutive Fibonacci numbers, that is, the convergents of the continued fraction of the golden number).

The foregoing examples seem simpler than the orbits on a triangle, and that is so not only because those maps are defined on subsets of the real line and we may follow their iterations on their graphs. Now we have no fewer than three maps - each corresponding to one side of the triangle - and, in each iteration, we can choose one of them at will. More precisely, if we denote by $\pi_{a}, \pi_{b}$, and $\pi_{c}$ the three orthogonal projections on the sides of a triangle $\mathcal{T}$, we may combine these maps in several ways. For instance, we may take:

(i) The map $\phi_{a b}: \mathbb{R}^{2} \rightarrow \mathbb{R}^{2}$, which assigns to each point $P$ in the plane its image after the composition of the two orthogonal projections $\pi_{a}$ and $\pi_{b}$.

(2i) The map $\phi_{a b c}: \mathbb{R}^{2} \rightarrow \mathbb{R}^{2}$, defined by the composition of the three projections $\pi_{a}, \pi_{b}$, and $\pi_{c}$, in this precise order.

(3i) The map $\phi_{b a c}: \mathbb{R}^{2} \rightarrow \mathbb{R}^{2}$, defined by the composition of the three projections $\pi_{b}, \pi_{a}$, and $\pi_{c}$, in this order.

(4i) A random sequence of letters from $\{a, b, c\}$, say $\lambda=$ $\left(\lambda_{n}\right)_{n \in \mathbb{N}}$, and the corresponding dynamics $\phi_{\lambda}$ whose iterates are given by

$$
\begin{aligned}
\left(\phi_{\lambda}\right)^{0} & =\text { Identity } \\
\left(\phi_{\lambda}\right)^{n} & =\pi_{\lambda_{n}} \circ \pi_{\lambda_{n-1}} \circ \cdots \circ \pi_{\lambda_{1}}
\end{aligned}
$$

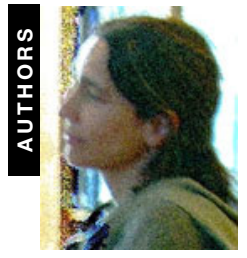

MARIA CARVALHO is a professor in the Department of Mathematics at the University of Porto. Her postgraduate studies in Ergodic Theory were completed at IMPA, in Rio de Janeiro, Brazil, under the guidance of Ricardo Mañé.

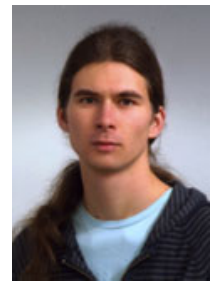

MIKA HAGER has Finnish origins and much appreciates northern forests. He started studying Architecture but soon became interested in logical games, and he even won a prize at a Portuguese Mathematical Olympiad. He has recently graduated in Mathematics from the University of Porto.

Departamento de Matemática

Faculdade de Ciências da Universidade do Porto

Rua do Campo Alegre, 687

4169-007 Porto

Portugal

e-mail: mpcarval@fc.up.pt

\author{
Departamento de Matemática \\ Faculdade de Ciências da Universidade \\ do Porto \\ Rua do Campo Alegre, 687 \\ 4l69-007 Porto \\ Portugal \\ e-mail: samuli.hager@gmail.com
}



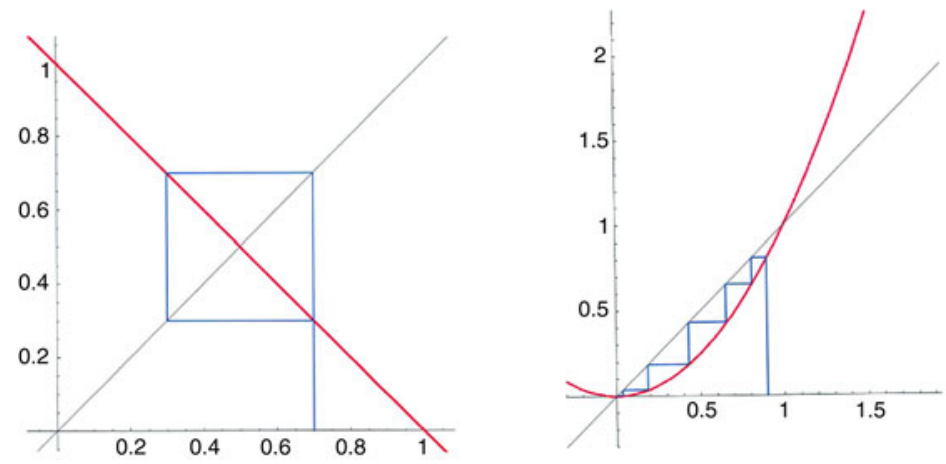

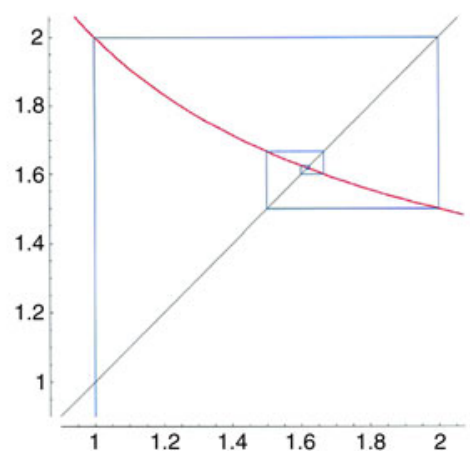

where, for each $Q \in \mathbb{R}^{2}$, the point $\pi_{\lambda_{j}}(Q)$ denotes the orthogonal projection of $Q$ on the side labelled by $\lambda_{j}$.

All these arrangements of the three maps give rise to an iterated function system (see [1]), because each projection is essentially a contraction on $\mathcal{T}$ - or rather, on a small compact extension $\mathcal{E}_{\mathcal{T}}$ of the sides of $\mathcal{T}$, since the orthogonal projections of one side on another may well fall outside $\mathcal{T}$. In fact, each map $\pi_{\tau}$ satisfies (denoting by $\|\cdot\|$ the usual Euclidean norm)

$$
\forall P, Q \in \mathbb{R}^{2} \quad\left\|\pi_{\tau}(P)-\pi_{\tau}(Q)\right\| \leq\|P-Q\|
$$

with equality if and only if the segment $P Q$ is parallel to the side labelled by $\tau$. Thus, if we start with two points $P$ and $Q$ on a side of $\mathcal{T}$, their orthogonal projections on the other sides of the triangle are strictly closer to each other. In general, we are dealing with contractions with rates no larger than $\kappa=$ $\max \{|\cos (\alpha)|,|\cos (\beta)|,|\cos (\gamma)|\}<1$, and, therefore, it is no surprise that we find global attractors in this context, although the geometrical properties they exhibit are quite astonishing.

\section{Orthogonal Projections}

Let $\mathcal{T}$ be a triangle with sides $a, b, c$ and angles $\alpha, \beta, \gamma$ (all different from zero and $\pi$ ), and $P$ a point in the plane. To simplify the calculations, we may use coordinates, assuming that $P=\left(P_{1}, P_{2}\right)$, the side $b$ lies on the $x$-axis and $a$ is on the line that goes through $(0,0)$ at an angle equal to $\gamma$. Then the points of the sequence $\left(\left(\phi_{a b}\right)^{n}(P)\right)_{n \in \mathbb{N}}$, where $\phi_{a b}=\pi_{b} \circ \pi_{a}$, lie on the $x$-axis and converge to $(0,0)$. In fact, if $\vec{a}=(\cos (\gamma), \sin (\gamma))$ is a unit vector generating the line that contains the side $a$, then

$$
\begin{aligned}
\phi_{a b}(P) & =\pi_{b} \circ \pi_{a}(P)=\pi_{b}(<P, \vec{a}>\vec{a}) \\
& =(<P, \vec{a}>\cos (\gamma), 0)
\end{aligned}
$$

where $<,>$ denotes the usual inner product in $\mathbb{R}^{2}$. Therefore,

$$
\begin{aligned}
\left(\phi_{a b}\right)^{2}(P) & =\left(<\phi_{a b}(P), \vec{a}>\cos (\gamma), 0\right) \\
& =(<(<P, \vec{a}>\cos (\gamma), 0), \vec{a}>\cos (\gamma), 0) \\
& =\left(\left(<P, \vec{a}>\cos ^{2}(\gamma)\right) \cos (\gamma), 0\right) \\
& =\cos ^{2}(\gamma) \phi_{a b}(P)
\end{aligned}
$$

and, in general,

$$
\left(\phi_{a b}\right)^{n}(P)=\cos ^{2(n-1)}(\gamma) \phi_{a b}(P) .
$$

Thus, if $\gamma=\frac{\pi}{2}$, then the sequence $\left(\left(\phi_{a b}\right)^{n}(P)\right)_{n \in \mathbb{N}}$ is constant and equal to $(0,0)$, the point of intersection of the sides $a$ and $b$; if $\gamma \in] 0, \frac{\pi}{2}\left[\right.$, then $\left(\left(\phi_{a b}\right)^{n}(P)\right)_{n \in \mathbb{N}}$ also converges to $(0,0)$, and this sequence essentially lies inside the angle defined by $\gamma$; the limit is again $(0,0)$ if $\gamma \in] \frac{\pi}{2}, \pi[$, although now the sequence $\left(\left(\phi_{a b}\right)^{n}(P)\right)_{n \in \mathbb{N}}$ mainly lies on the complement of this angle. Similar conclusions are valid for the pairs $\{b, c\}$ and $\{a, c\}$.

We now proceed considering the three projections together, starting with the simplest case.

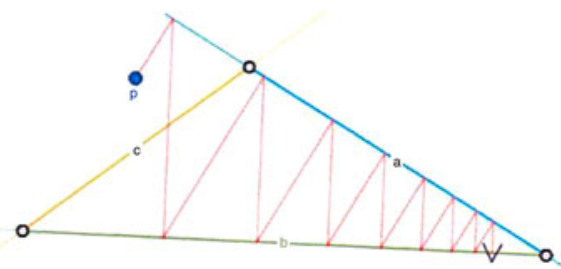

\section{Right Triangles}

As before, we position the triangle in a favourable corner: the side $a$ lies on the $x$-axis (so it is generated by the vector $(1,0)), b$ on the $y$-axis (generated by $(0,1)$ ), and the side $c$ lies on the line that passes through the point $\left(0, Y_{0}\right)$ with direction given by the vector $(-\cos (\beta), \sin (\beta))$, where $Y_{0}=|b|=|a| \tan (\beta) .^{3}$

Given $P=\left(P_{1}, P_{2}\right)$, we have

$$
\begin{aligned}
\phi_{a b c}(P) & =\pi_{c} \circ \pi_{b} \circ \pi_{a}(P)=\pi_{c} \circ \pi_{b}\left(P_{1}, 0\right)=\pi_{c}(0,0) \\
& =Y_{0} \cos (\beta)(\sin (\beta), \cos (\beta)) \\
& =|a| \sin (\beta)(\sin (\beta), \cos (\beta)),
\end{aligned}
$$

a point, say $\mathfrak{F}_{a b c}$, that lies on the side $c$ and is independent of $P$. It is the $\omega$-limit of any point $P$ of the plane by the dynamics $\phi_{a b c}$.

Let $A, B$, and $C$ denote the vertices of $\mathcal{T}$ (with right angle $\angle(A C B)$ ) and $A^{\prime}=C=(0,0), B^{\prime}=\mathfrak{F}_{a b c}$ and $C^{\prime}=$ $\pi_{a}\left(\mathfrak{F}_{a b c}\right)=\left(|a|(\sin (\beta))^{2}, 0\right)$ the three points of the orbit of $\mathfrak{F}_{a b c}$. The latter are the vertices of a new triangle $\mathcal{T}_{1}$ we will now compare with $\mathcal{T}$.

Since we are dealing with orthogonal projections, the angle $\angle\left(A^{\prime} C^{\prime} B^{\prime}\right)=\frac{\pi}{2}$, and so $\angle\left(A^{\prime} C^{\prime} B^{\prime}\right)=\angle(A C B)$. Moreover, $\angle\left(B^{\prime} A^{\prime} C^{\prime}\right)=\angle\left(B^{\prime} A^{\prime} B\right)=\alpha$, and therefore the triangles $\mathcal{T}$ and $\mathcal{T}_{1}$ are similar. In fact, $\mathcal{T}_{1}$ is the image of $\mathcal{T}$

\footnotetext{
${ }^{3}$ The figures illustrating the following pages were drawn by Manuel Arala Chaves (Mathematics Department, University of Porto).
} 


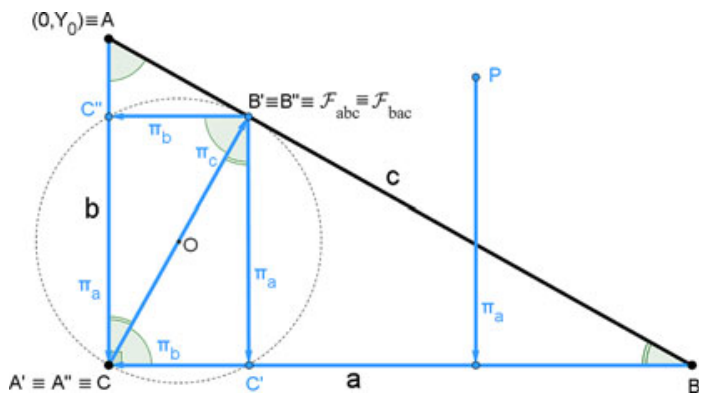

by a homothety with ratio $\frac{\left|A^{\prime} C^{\prime}\right|}{|A C|}$, that is, $\frac{|a| \sin ^{2}(\beta)}{|a| \tan (\beta)}=$ $\sin (\beta) \cos (\beta)$, followed by a 90 degree rotation. Moreover, the quotient of the areas of $\mathcal{T}_{1}$ and $\mathcal{T}$ is equal to

$$
\frac{\left|A^{\prime} C^{\prime}\right|^{2}}{|A C|^{2}}=(\sin (\beta) \cos (\beta))^{2} \text {. }
$$

which only depends on the angles of $\mathcal{T}$ and attains a maximum value, equal to $\frac{1}{4}$, when $\beta=\frac{\pi}{4}$, that is to say, when $\mathcal{T}$ is isosceles.

If we change the order in which the sides of $\mathcal{T}$ are visited, from $a b c$ to $b a c$, then a similar argument proves that $\phi_{b a c}$ also has a global attracting fixed point, say $\mathfrak{F}_{b a c}$, in the side $c$ of the triangle, which in fact coincides with $\mathfrak{F}_{a b c}$. However, its images by $\pi_{b}$ and $\pi_{a} \circ \pi_{b}$ build up a different limit triangle, say $\mathcal{T}_{2}$, whose vertices are $A^{\prime \prime}=C=(0,0)$, $B^{\prime \prime}=B^{\prime}=\mathfrak{F}_{a b c}$, and $C^{\prime \prime}=\pi_{b}\left(\mathfrak{F}_{a b c}\right)=(0,|a| \sin (\beta) \cos (\beta))$.

The triangle $\mathcal{T}_{2}$ is similar to $\mathcal{T}$, and so is similar to $\mathcal{T}_{1}$. Moreover, $\mathcal{T}_{1}$ and $\mathcal{T}_{2}$ have one side in common, $A^{\prime} B^{\prime}$ of the former, $A^{\prime \prime} B^{\prime \prime}$ of the latter; thus $\mathcal{T}_{1}$ and $\mathcal{T}_{2}$ are congruent. And the rotation by 180 degrees around $\mathcal{O}=\frac{1}{2} \mathfrak{F}_{a b c}$ sends one onto the other. Finally, observe that, as $\mathcal{O}$ is the midpoint of the hypotenuses of both $\mathcal{T}_{1}$ and $\mathcal{T}_{2}$, it is also the common circumcenter of both triangles - so, the six vertices lie on the same circle.

\section{General Case}

Let now $\mathcal{T}$ be any triangle with vertices $A, B$, $C$, sides $a, b, c$, and opposite angles $\alpha, \beta, \gamma$. As before, we may position it so that $A=(0,0)$, the side $a=A B$ passes through $(0,0)$ with slope determined by $\beta$, the side $c=A C$ lies on the $x$-axis, and $b=B C$ is on the line that crosses the $y$-axis at the point $\left(0, Y_{0}\right)$, where $Y_{0}=|c| \tan (\alpha)$.

If $\vec{a}$ and $\vec{b}$ denote unit vectors in the direction of the side $a$ (that is, $\vec{a}=(\cos (\beta), \sin (\beta)))$, and the side $b$ (that is, $\vec{b}=(-\cos (\alpha), \sin (\alpha)))$, respectively, then, given a point $P=\left(P_{1}, P_{2}\right)$ in the plane, we have

$$
\begin{aligned}
\phi_{a b c}(P)= & \pi_{c} \circ \pi_{b} \circ \pi_{a}(P)=\pi_{c} \circ \pi_{b}(<P, \vec{a}>\vec{a}) \\
= & \pi_{c}\left[\left(Y_{0} \sin (\alpha) \cos (\alpha), Y_{0}(\cos (\alpha))^{2}\right)\right. \\
& \quad+<P, \vec{a}><\vec{a}, \vec{b}>\vec{b}] \\
= & \left(Y_{0} \sin (\alpha) \cos (\alpha)-<P, \vec{a}><\vec{a}, \vec{b}>\cos (\alpha), 0\right) \\
= & \left(Y_{0} \sin (\alpha) \cos (\alpha)-\left[P_{1} \cos (\beta)+P_{2} \sin (\beta)\right]\right. \\
& \quad \times \cos (\alpha) \cos (\gamma), 0) .
\end{aligned}
$$

If we restrict $\phi_{a b c}$ to the line that contains the side $c$ - which is the $x$-axis - we are reduced to the analysis of the onedimensional dynamics

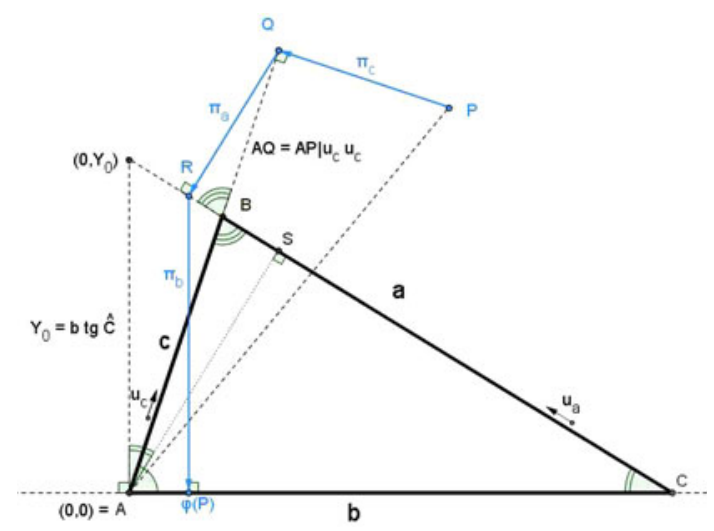

$$
\varphi: x \in \mathbb{R} \rightarrow Y_{0} \sin (\alpha) \cos (\alpha)-x \cos (\alpha) \cos (\beta) \cos (\gamma) .
$$

The equation $\phi_{a b c}(P)=P($ or equivalently $\varphi(x)=x)$ may now be solved to reveal a unique fixed point, $\mathcal{G}_{a b c}$, with coordinates

$$
\begin{aligned}
& \mathcal{G}_{1}=\frac{Y_{0} \sin (\alpha) \cos (\alpha)}{1+\cos (\alpha) \cos (\beta) \cos (\gamma)} \\
& \mathcal{G}_{2}=0 .
\end{aligned}
$$

Moreover, $\mathcal{G}_{a b c}$ is a global attractor. To prove this, it is enough to verify that $\varphi$ is a contraction. Given $x \in \mathbb{R}$, by the Mean-Value Theorem, there exists some $\xi$ such that

$\varphi(x)-\mathcal{G}_{1}=\varphi^{\prime}(\xi)\left[x-\mathcal{G}_{1}\right]=-\cos (\alpha) \cos (\beta) \cos (\gamma)\left[x-\mathcal{G}_{1}\right]$

which is zero, as expected, in the case of a right triangle. If the triangle is not right, we proceed using Lagrange multipliers to find the extremes of the derivative given by $-\cos (\alpha) \cos (\beta) \cos (\gamma)$. Consider the realvalued map $f:(x, y, z) \rightarrow-\cos (x) \cos (y) \cos (z)$ restricted to the set $\left\{(x, y, z) \in\left(\mathbb{R}^{+}\right)^{3}: x+y+z=\pi\right\}$. Take $g:(x, y, z) \rightarrow x+y+z-\pi$ and the gradients

$$
\nabla_{g}=\left(\begin{array}{l}
1 \\
1 \\
1
\end{array}\right) \text { and } \nabla f=\left(\begin{array}{c}
\sin (x) \cos (y) \cos (z) \\
\cos (x) \sin (y) \cos (z) \\
\cos (x) \cos (y) \sin (z)
\end{array}\right) \text {. }
$$

Then the equality $\nabla f=\mu \nabla g$ is equivalent to $\tan (x)=$ $\tan (y)=\tan (z)$, which can only happen if either $\pi \in$ $\{x, y, z\}$ or $x=y=z=\frac{\pi}{3}$. The map $f$ attains a minimum, equal to $-\frac{1}{8}$, in the latter case, and a maximum, equal to 1 , in the former. But, since within the triangles no angle can be equal to $\pi$, we may guarantee that $-\frac{1}{8} \leq \cos (\alpha) \cos (\beta) \cos (\gamma)<1$. Therefore, for any point $P$, the sequence $\left(\left(\phi_{a b c}\right)^{n}(P)\right)_{n \in \mathbb{N}}$ converges to $\mathcal{G}_{a b c}$.

Concerning the shape and position of the triangle $\mathcal{T}_{1}$ with vertices $A^{\prime}=\mathcal{G}_{a b c}, B^{\prime}=\pi_{a}\left(\mathcal{G}_{a b c}\right)$ and $C^{\prime}=\pi_{b} \circ \pi_{a}\left(\mathcal{G}_{a b c}\right)$, we recall that $\angle A C B=\alpha, \angle B A C=\beta, \angle A B C=\gamma$ and, moreover, that the line $A^{\prime} C^{\prime}$ is vertical, the line $B^{\prime} C^{\prime}$ is orthogonal to the side $b$, and the line $A^{\prime} B^{\prime}$ is orthogonal to $a$. Therefore $\angle A^{\prime} C^{\prime} B^{\prime}=\alpha, \angle B^{\prime} A^{\prime} C^{\prime}=\beta$, and $\angle C^{\prime} B^{\prime} A^{\prime}=\gamma$, which means that the triangle $\mathcal{T}_{1}$ is similar to the original one but is rotated by 90 degrees. Furthermore, the areas of $\mathcal{T}_{1}$ and $\mathcal{T}$ have ratio equal to 


$$
\frac{\left|A^{\prime} C^{\prime}\right|^{2}}{|A C|^{2}}=\left[\frac{\sin (\alpha) \cos (\alpha)+\sin (\alpha) \cos (\beta) \cos (\gamma)}{1+\cos (\alpha) \cos (\beta) \cos (\gamma)}\right]^{2},
$$

which only depends on the angles of $\mathcal{T}$.

\section{Changing the Order}

Analogous calculations show that $\phi_{\text {bac }}$ has a global attracting fixed point, too, say $\mathcal{G}_{b a c}$, in the side $c$ of the triangle $\mathcal{T}$, given by

$$
\mathcal{G}_{b a c}=\left(\frac{Y_{0} \cos (\alpha) \cos (\beta) \sin (\gamma)}{1+\cos (\alpha) \cos (\beta) \cos (\gamma)}, 0\right) .
$$

(If the triangle is right, with $\beta=\frac{\pi}{2}$, then this fixed point is $(0,0)$, as expected.) The point $\mathcal{G}_{b a c}$ and its first iterates by $\pi_{b}$ and $\pi_{a} \circ \pi_{b}$ build up a triangle $\mathcal{T}_{2}$, similar to $\mathcal{T}$, which is congruent to $\mathcal{T}_{1}$ but rotated by 180 degrees. In fact, the sides of $\mathcal{T}_{1}$ are pairwise parallel to those of $\mathcal{T}_{2}$ and, moreover,

$$
\left\|A^{\prime}-B^{\prime}\right\|=\left\|A^{\prime \prime}-B^{\prime \prime}\right\|=\frac{Y_{0} \cos (\alpha) \sin (\beta) \sin (\gamma)}{1+\cos (\alpha) \cos (\beta) \cos (\gamma)} .
$$

The common circumcenter is the point of intersection of the diagonals of the rectangle with vertices $A^{\prime}, B^{\prime}, A^{\prime \prime}, B^{\prime \prime}$, and so it is the midpoint of the segments $A^{\prime} A^{\prime \prime}$ and $B^{\prime} B^{\prime \prime}$.

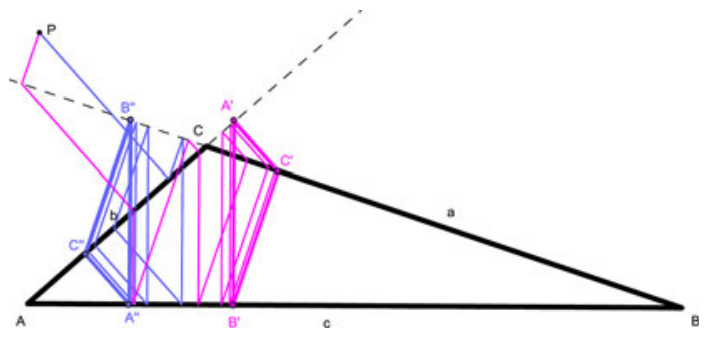

\section{Other Periodic Arrangements}

The iteration of $\phi_{a b}$ is related to the choice of the periodic sequence $a b a b a b \ldots$, with period 2, in the space of letters $\{a, b, c\}^{\mathbb{N}}$. As we verified, the global attractor this dynamic presents - the intersection of the sides $a$ and $b$ - is a fixed point (with period two if seen distinctly by $a$ and $b$ ). Analogously, $\phi_{a b c}$ and $\phi_{b a c}$ correspond to choices of periodthree sequences, namely $a b c a b c . .$. and $b a c b a c \ldots$, and the global attractor they exhibit is a fixed point on $c$ whose projections on the other sides of $\mathcal{T}$ build up a period-three orbit for the iteration of those projections in the specified order. We may conjecture that, for any other $k$-periodic sequence $\lambda=\left(\lambda_{1} \ldots \lambda_{k} \lambda_{1} \ldots \lambda_{k} \ldots\right)$ in $\{a, b, c\}^{\mathbb{N}}$, the associated dynamical system $\phi_{\lambda}$ has a globally attracting fixed point whose images by all $\pi_{\lambda}$ s make up a polygon with a number of sides somehow determined by $k$ (some of these $k$ points may coincide, as happens with the combination $a b c b$ on a right triangle with legs $a$ and $b$ ).

The proof of this conjecture is similar to the one described previously for $a b c a b c \ldots$. The key reason why this property holds is the already mentioned fact that, given a finite block of letters from $a, b, c$, with minimum period $k$ and no two equal consecutive letters (a redundancy we may discard in advance), say $\tau_{1} \tau_{2} \cdots \tau_{k}$, the map $\phi_{\tau_{1} \tau_{2} \cdots \tau_{k}}$ is a contraction on $\mathcal{E}_{\mathcal{T}}$ at a rate better than or equal to $\kappa$. Moreover, as a consequence of Theorems 3.7.1 and 4.2.1 of [1], the set of periodic orbits of the iterated function system $\phi$ is dense in $\mathcal{T}$.

\section{Nonperiodic $\Omega$-Limits}

What happens if we choose a nonperiodic sequence $\lambda=\left(\lambda_{n}\right)_{n \in \mathbb{N}}$ in $\{a, b, c\}^{\mathbb{N}}$ ? If, although not being periodic, it uses only two of the three possible letters, then there are no novelties: the $\omega$-limit of each orbit is the intersection point of the two lines that contain the chosen sides of $\mathcal{T}$, although the convergence may be slower than that detected for $a b a b . .$. However, if $\lambda$ uses all the three letters and is random enough, then the asymptotic behaviour may change from the periodic pattern. For instance, we may find a sequence $\lambda=\left(\lambda_{n}\right)_{n \in \mathbb{N}}$ in $\{a, b, c\}^{\mathbb{N}}$ such that the dynamics of $\phi_{\lambda}$ has a dense orbit on $\mathcal{T}$ in the following way.

(I) For any pair of points $P$ and $Q$ in $\mathcal{T}$ and any neighbourhood $\mathcal{I}$ of $Q$ in $\mathcal{T}$, we can choose a finite block $r$ of letters in $\{a, b, c\}^{\mathbb{N}}$ and, starting at $P$, reach $\mathcal{I}$ by iterating $\phi_{r}$

Take an $\epsilon>0$, points $P, Q \in \mathcal{T}$ and an open segment $\mathcal{I}$ on the side of $\mathcal{T}$ where $Q$ is, containing $Q$ and with length smaller than $\epsilon$ (this arc may end at a vertex of $\mathcal{T}$ if $Q$ is a vertex). As mentioned previously, there is a point $R \in \mathcal{I}$ that belongs to a global attracting periodic orbit of period $p$ associated to the dynamics of a map $\phi_{r}=\pi_{r_{p}} \circ \pi_{r_{p-1}} \circ \cdots \circ$ $\pi_{r_{1}}$. Then, iterating the map $\phi_{r}$ a large enough number $N_{P, Q, \epsilon}$ of times, the orbit of $P$ by $\phi_{r}$ comes $\epsilon$-close to $R$, and so to $Q$.

(II) For any point $P \in \mathcal{T}$, we may find a sequence $r$ of letters in $\{a, b, c\}^{\mathbb{N}}$ such that the orbit of $P$ by $\phi_{r}$ visits every neighbourbood in $\mathcal{T}$.

Fix a point $P \in \mathcal{T}$ and a positive $\epsilon$, and let $\mathcal{D}=$ $\left\{D_{1}, D_{2}, \ldots D_{k}, \ldots\right\}$ be a countable dense subset of $\mathcal{T}$. By (I), there are $N_{1}=N_{P, D_{1}, \epsilon} \in \mathbb{N}$ and a finite choice $r_{11}, \ldots, r_{1 N_{1}}$, such that the distance between $D_{1}$ and the point

$$
R_{1}=\pi_{r_{1 N_{1}}} \circ \cdots \circ \pi_{r_{11}}(P)
$$

is less than $\epsilon$. Similarly, there are $N_{2}=N_{R_{1}, D_{2}, \epsilon} \in \mathbb{N}$ and a finite choice $r_{21}, \ldots, r_{2 N_{2}}$, such that the distance between $D_{2}$ and the point

$$
R_{2}=\pi_{r_{2 N_{2}}} \circ \cdots \circ \pi_{r_{21}}\left(R_{1}\right)
$$

is less than $\epsilon$. Thus, the distance between $D_{2}$ and the point

$$
R_{2}=\pi_{r_{2 N_{2}}} \circ \cdots \circ \pi_{r_{21}} \circ \pi_{r_{1 N_{1}}} \circ \cdots \circ \pi_{r_{11}}(P)
$$

is also smaller than $\epsilon$. Proceeding recursively, we find a sequence $r$, made up of the countable union of the finite blocks of the previous $r_{i j}$ 's, such that, by the corresponding $\phi_{r}$, the point $P$ comes $\epsilon$-near to all points of $\mathcal{D}$, and therefore $\epsilon$-close to any point in $\mathcal{T}$.

(III) There is a random enough sequence of letters whose corresponding dynamics has a dense orbit.

Fix again $P \in \mathcal{T}$ and $\mathcal{D}$ as previously mentioned, and take $\epsilon=1$. As $\mathcal{T}$ is compact, there is a finite subcovering by 
triangle-segments $\left.\mathcal{I}_{i, 1}=\right] D_{i}-1, D_{i}+1[\cap \mathcal{T}$, where $i$ belongs to some set $\left\{k_{11}, \ldots, k_{12}\right\}$. Apply now the previous argument to obtain a finite block $t_{11} t_{12} \cdots t_{1 \mu_{1}}$ of letters in $\{a, b, c\}$ such that the set of iterates

$$
\left\{\pi_{t_{1 \mu_{1}}} \circ \cdots \circ \pi_{t_{11}}(P), \cdots \cdots, \pi_{t_{21}} \circ \pi_{t_{11}}(P), \pi_{t_{11}}(P)\right\}
$$

intersects all the $\operatorname{arcs} \mathcal{I}_{i, 1}$.

Repeat the procedure with $\epsilon=\frac{1}{2}$ and the subcovering $\left.\mathcal{I}_{i, \frac{1}{2}}=\right] D_{i}-\frac{1}{2}, D_{i}+\frac{1}{2}[\cap \mathcal{T}$, where $i$ belongs to some set $\left\{k_{21}, \ldots, k_{22}\right\}$. Consider the point $P_{\mu_{1}}=\pi_{t_{1 \mu_{1}}} \circ \cdots \circ \pi_{t_{11}}(P)$. By a similar reasoning, there exists a finite block $t_{21}$ $t_{22} \cdots t_{2 \mu_{2}}$ of $\{a, b, c\}$ such that the set of iterates

$$
\left\{\pi_{t_{2 \mu_{2}}} \circ \cdots \circ \pi_{t_{21}}\left(P_{\mu_{1}}\right), \cdots \cdots, \pi_{t_{22}} \circ \pi_{t_{21}}\left(P_{\mu_{1}}\right), \pi_{t_{21}}\left(P_{\mu_{1}}\right)\right\}
$$

intersects all the $\operatorname{arcs} \mathcal{I}_{i, \frac{1}{2}}$.

In general, for any natural $j>1$, take $\epsilon=\frac{1}{j}$, a subcovering $\left.\mathcal{I}_{i, \frac{1}{j}}=\right] D_{i}-\frac{1}{j}, D_{i}+\frac{1}{j}[\cap \mathcal{T}$, where $i$ belongs to some set $\left\{k_{j 1}, \ldots, k_{j 2}\right\}$, the previously defined point $P_{\mu_{j-1}}$ and a finite block $t_{j 1} t_{j 2} \cdots t_{j \mu_{l}}$ of $\{a, b, c\}$ such that the set of iterates

$$
\left\{\pi_{t_{j \mu_{l}}} \circ \cdots \circ \pi_{t_{j 1}}\left(P_{\mu_{j-1}}\right), \cdots \cdots, \pi_{t_{j 2}} \circ \pi_{t_{j 1}}\left(P_{\mu_{j-1}}\right), \pi_{t_{j 1}}\left(P_{\mu_{j-1}}\right)\right\}
$$

intersects all the $\operatorname{arcs} \mathcal{I}_{i, \frac{1}{j}}$. If $\lambda \in\{a, b, c\}^{\mathbb{N}}$ is defined by gluing together all these finite blocks in the order determined by increasing $j$, then the orbit $\left(\left(\phi_{\lambda}\right)^{n}(P)\right)_{n \in \mathbb{N}}$ is dense in $\mathcal{T}$.

Moreover,

(IV) The dynamics $\left(\left(\phi_{\lambda}\right)^{n}\right)_{n \in \mathbb{N}}$ on $\mathcal{T}$ is minimal.

Consider $P \in \mathcal{T}$ and the sequence $\lambda \in\{a, b, c\}^{\mathbb{N}}$ obtained in (III) for $P$, so that the orbit of $P$ by $\phi_{\lambda}$ is dense in $\mathcal{T}$. If we take another point $X \in \mathcal{T}$, we may find an iterate of $P$ arbitrarily close to $X$. Moreover, from this moment on, each projection contracts distances, so the orbit of $X$ by $\phi_{\lambda}$ shadows that of $P$ and, therefore, is dense, too.

\section{Generalizations}

If we consider another angle to project, or three different angles, one for each side of $\mathcal{T}$, not all the above-mentioned properties manage to survive.

\section{Nonorthogonal Projections}

Given an angle $\theta \in]-\frac{\pi}{2}, \frac{\pi}{2}\left[\right.$, let $\pi_{d}^{\theta}$ denote the projection on the side labelled by $d$ along a line that makes with the orthogonal direction to that side an oriented angle equal to $\theta$. When $\theta=0$ (or very close to 0 ), we go back to the setting of the first section. Consider then, for a choice $\lambda=\left(\lambda_{n}\right)_{n \in \mathbb{N}}$ in $\{a, b, c\}^{\mathbb{N}}$, the dynamics defined by

$$
\begin{aligned}
& \left(\psi_{\lambda}^{\theta}\right)^{0}=\text { Identity } \\
& \left(\psi_{\lambda}^{\theta}\right)^{n}=\pi_{\lambda_{n}}^{\theta} \circ \pi_{\lambda_{n-1}}^{\theta} \circ \cdots \circ \pi_{\lambda_{1}}^{\theta} .
\end{aligned}
$$

We detect two caveats: The existence of a global attractor for $\psi_{a b}^{\theta}, \psi_{a b c}^{\theta}$ and $\psi_{b a c}^{\theta}$ is no longer guaranteed, because $\pi_{d}^{\theta}$ may fail to contract distances; and the calculations become nastier and explicit expressions of the possible fixed point and its derivative are rather hard to obtain. So let us just sketch the main procedure to deal with this case; further analysis may be performed numerically for definite values of $\theta$.
Label the elements of the triangle, and position them favourably as we did before. Consider a point $P=\left(P_{1}, P_{2}\right)$ in the plane and the vectors

$$
\begin{aligned}
\vec{a}= & (\cos (\beta), \sin (\beta)) \\
& : \text { a generator of the line that contains the side } a \\
\vec{o}= & (\sin (\beta),-\cos (\beta)) \\
& : \text { a generator of the orthogonal line to the side } a \\
\vec{v}= & (\sin (\beta-\theta),-\cos (\beta-\theta)) \\
& : \text { a generator of the line with slope } \theta \text { from } \vec{o} .
\end{aligned}
$$

Then $\pi_{a}^{\theta}(P)$ is the intersection of the lines $\{t \vec{a}: t \in \mathbb{R}\}$ and $\{P+t \vec{v}: t \in \mathbb{R}\}$. Straightforward calculations show that $\pi_{a}^{\theta}(P)$ has coordinates

$$
P_{1}+\left[\frac{P_{2} \cos (\beta)-P_{1} \sin (\beta)}{\cos (\theta)}\right] \sin (\beta-\theta)
$$

and

$$
P_{2}-\left[\frac{P_{2} \cos (\beta)-P_{1} \sin (\beta)}{\cos (\theta)}\right] \cos (\beta-\theta) .
$$

Assume, for the moment, that the side $b$ lies on the $x$-axis. Then $\pi_{b}^{\theta} \circ \pi_{a}^{\theta}(P)$ is realized through the intersection of the lines $\{(t, 0): t \in \mathbb{R}\}$ and $\left\{\pi_{a}^{\theta}(P)+t \vec{\theta}: t \in \mathbb{R}\right\}$, where $\vec{\theta}=$ $(-\sin (\theta),-\cos (\theta))$ is a vector that makes an angle $\theta$ with the perpendicular line to the side $b$. So, in this special position of the two sides $a$ and $b$, the second coordinate of $\psi_{a b}^{\theta}\left(P_{1}, P_{2}\right)$ is zero, and the first is given by

$$
\begin{aligned}
P_{1} & +\left[\frac{P_{2} \cos (\beta)-P_{1} \sin (\beta)}{\cos (\theta)}\right] \sin (\beta-\theta)- \\
& -\tan (\theta)\left(P_{2}-\left[\frac{P_{2} \cos (\beta)-P_{1} \sin (\beta)}{\cos (\theta)}\right] \cos (\beta-\theta)\right) .
\end{aligned}
$$

Hence, $\psi_{d}^{\theta}(0,0)=(0,0)$ and the derivative along the direction $b$ at $(0,0)$ of $\psi_{a b}^{\theta}$ is equal to

$$
1-\frac{\sin ^{2}(\beta)}{\cos ^{2}(\theta)}
$$

an expression whose absolute value may fail to be less than 1. (Check, for instance, what happens when $\beta=\frac{\pi}{2}$ and $\theta>\frac{\pi}{4}$.)

By a similar vectorial analysis, we may determine $\pi_{b}^{\theta} \pi_{a}^{\theta}(P)$ in a more general position. We then deduce that if $\psi_{a b c}^{\theta}$ has a globally attracting fixed point $\left(\right.$ say $\left.\mathcal{H}_{a b c}=\left(\mathcal{H}_{1}, 0\right)\right)$ at the line that contains the side $c$, then it is a vertex of a triangle $\mathcal{S}_{1}$ similar to $\mathcal{T}$ but rotated through an angle of $90-\theta$ degrees, whose other vertices are the images of $\mathcal{H}_{a b c}$ by the projections $\pi_{b}^{\theta}$, and $\pi_{a}^{\theta}$. Moreover, if $\mathcal{T}$ has angles $\alpha, \beta$, and $\gamma$, and $|c|$ denotes the length of the side $c$, then the quotient of the areas of $\mathcal{S}_{1}$ and $\mathcal{T}$ is equal to

$$
\frac{\text { area of } \mathcal{S}_{1}}{\text { area of } \mathcal{T}}=\left(\frac{|c|-\mathcal{H}_{1}}{|c|[\sin (\theta)+\cot (\alpha) \cos (\theta)]}\right)^{2} \text {. }
$$

Unsurprisingly, $\psi_{b a c}^{\theta}$ has a fixed point, too, which is a vertex of a triangle $\mathcal{S}_{2}$ congruent to $\mathcal{S}_{1}$, and each triangle is the image of the other by a rotation of 180 degrees around the common circumcenter. 


\section{Different Angles of Projection}

We get different results if the angles of projection vary with the sides of the triangle. One can adapt the previous calculations to three different angles of projection, say $\theta_{1}, \theta_{2}$, and $\theta_{3}$, belonging to $]-\frac{\pi}{2}, \frac{\pi}{2}[$. As expected, the explicit formulas are more difficult to unravel. Numerical experiments indicate that, although there still may be a global attracting fixed point at the side $c$, the distortion may be too strong, and the limit-triangle no longer inherits significant traits of the original one.

Indeed, if the triangle $\mathcal{T}$ has sides $a, b, c$, and angles $\alpha, \beta, \gamma$, and each point in the plane travels along lines that intersect $\mathcal{T}$ at angles $\theta_{1}, \theta_{2}$, and $\theta_{3}$, respectively, then the angles of the limit triangle $\mathcal{T}_{1}$ for $\psi_{a b c}^{\theta_{1} \theta_{2} \theta_{3}}$, if it exists, are $\alpha+\theta_{2}-\theta_{3}, \beta+\theta_{3}-\theta_{1}$, and $\gamma+\theta_{1}-\theta_{2}$. If $\mathcal{T}_{1}$ does not degenerate, there seems to exist also a limit triangle $\mathcal{T}_{2}$ for $\psi_{b a c}^{\theta_{2} \theta_{1} \theta_{3}}$, which is congruent to $\mathcal{T}_{1}$ and whose sides are pairwise parallel to those of $\mathcal{T}_{1}$.

\section{To Know More}

We leave the reader with two of many questions that we have not addressed in this article:
1. May we generalize most of these properties when other polygons replace the triangle? For instance, being a parallelogram is preserved under this type of dynamics. What else?

2. What is the asymptotic behaviour if, instead of the plane, the projections act on a 2-sphere (where two similar triangles are congruent), a nonflat 2-torus, or the Poincaré disk?
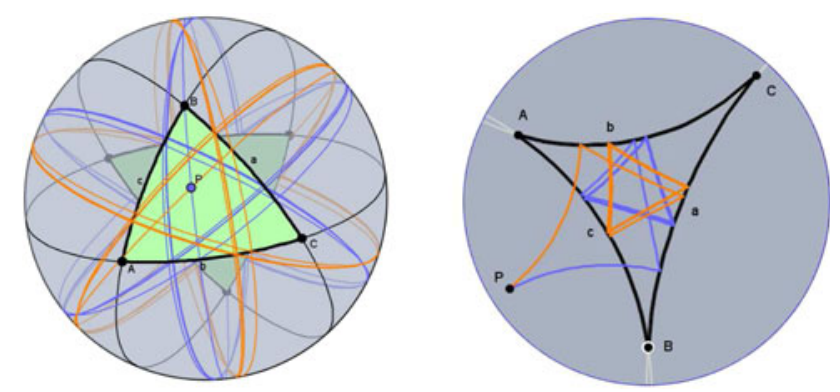

\section{REFERENCE}

1. M. Barnsley, Fractals Everywhere, Academic Press, 1988. 